Dass unser Beruf - heute noch mehr als in früheren Zeiten - zu einer schwerwiegenden Erschöpfung führen kann, ist in der Bevölkerung und bei den Behörden noch überhaupt nicht anerkannt. Und auch viele Kolleginnen und Kollegen weisen diese Tatsache weit von sich. Doch diese Erschöpfungszustände haben gravierende Auswirkungen auf die Gesundheit der betroffenen Ärztinnen und Ärzte und auch auf ihre berufliche Leistungsfähigkeit.
Im nachstehenden Editorial beschreibt Martina Hersperger, Leiterin der Abteilung DDQ der $\mathrm{FMH}$, ein bemerkenswertes Projekt, das uns sehr am Herzen liegt. Hoffentlich werden jene Ärztinnen und Ärzte, die leider eine solche Situation durchleben, das Angebot nutzen, das sich ihnen bietet.

Dr. med. Jacques de Haller, Präsident der FMH

\title{
ReMed - Unterstützung zum richtigen Zeitpunkt
}

Überlastung, Sucht oder Burn-out: Krisen haben viele Gesichter. Und sie bleiben nicht ohne Auswirkungen auf die berufliche Arbeit - das ist bei Ärztinnen und Ärzten nicht anders als bei anderen Berufsgruppen: Wer psychisch und physisch angeschlagen ist, kann sich weniger intensiv und empathisch um seine Patienten kümmern. Die Gesundheit der Ärzteschaft ist der FMH deshalb ein besonders zentrales Anliegen. 2007 haben wir mit ReMed (www.swiss-remed.ch) ein Unterstützungsnetzwerk initiiert, das sich ganz auf dieses wichtige Thema konzentriert. Ziel ist es, mit gesunden und zufriedenen Ärztinnen und Ärzten eine qualitativ hochstehende und sichere medizinische Versorgung der Bevölkerung zu gewährleisten.

ReMed berät Ärztinnen und Ärzte in Krisen. Bei jeder Kontaktaufnahme meldet sich innerhalb von maximal 72 Stunden ein erfahrenes Beraterteam und steht unterstützend zur Seite. Die Beraterinnen und Berater - ebenfalls Ärzte - zeigen individuelle Handlungsmöglichkeiten auf und suchen gemeinsam mit dem Hilfesuchenden nach Wegen aus der Krise. In der dreijährigen Pilotphase fokussierte

\section{Auch Ärztinnen und Ärzte können aus dem Gleichgewicht geraten}

sich ReMed zunächst auf die Kantone Thurgau und Neuenburg. Doch rasch meldeten sich Ärztinnen und Ärzte aus der ganzen Schweiz beim Beraterteam. Über 80 Mal ist das Unterstützungsnetzwerk seither aktiv geworden. Das zeigt uns, dass wir mit ReMed den richtigen Weg eingeschlagen haben und eine Vertrauensbasis schaffen konnten. Für den Zentralvorstand der FMH war deshalb klar, ReMed nach Abschluss der Pilotphase in der ganzen Schweiz weiterzuführen und zu institutionalisieren. Am letzten Freitag erfolgte nun die schweizweite Lancierung von ReMed. Die FMH übernimmt als Trägerin weiterhin die Finanzierung, die Verantwortung liegt immer noch beim Leitungsausschuss von ReMed. In einem neu gegründeten Beirat sind Dach- und Partnerorganisationen der Ärzteschaft vertreten - ein Zeichen, dass das Unterstützungsnetzwerk breit abgestützt ist.

Wer sich an ReMed wendet, geniesst die gleichen Rechte wie ein Patient- nichts geschieht ohne Zustimmung des Ratsuchenden, alles, was besprochen wird, bleibt vertraulich.

\section{ReMed hilft bei Krisen}

Da ReMed an das ärztliche Berufsgeheimnis gebunden ist, hat die FMH zu keinem Zeitpunkt Einblick in die Personenund Betreuungsakten: Ärztliche und organisatorische Aufgaben sind klar getrennt.

Damit sich Ärztinnen und Ärzte in Krisensituationen an ReMed wenden, müssen sie das Angebot unbedingt kennen. Das Unterstützungsnetzwerk hat deshalb ein neues Erscheinungsbild erhalten. Wir freuen uns, dass die renommierte Basler Karikaturistin ANNA - Leserinnen und Lesern der SÄZ bestens bekannt - dazu einen Cartoon geschaffen hat, der die Idee hinter ReMed bestens trifft. Am einfachsten überzeugen Sie sich selbst auf Seite 1721 dieser Ausgabe. Sie finden dort auch ein informatives Interview mit dem Programmleiter von ReMed, dem Allgemeinmediziner Dr. med. Michael Peltenburg.

Zudem haben wir ein umfangreiches Informationspaket geschnürt: In den nächsten Tagen erhalten die Ärztegesellschaften der FMH, viele Spitäler und grosse Gruppenpraxen eine Sendung mit Flyern und Kleinplakaten, die das Angebot von ReMed kurz und anschaulich erläutern. Werden diese Unterlagen dort plaziert, wo Ärztinnen und Ärzte auch hinschauen - zum Beispiel im Pausenraum oder in der Garderobe - können wir für noch mehr Ratsuchende eine Anlaufstelle sein. Wir zählen dabei auf Ihre Mithilfe und danken schon jetzt für Ihre Unterstützung.

Martina Hersperger,

Abteilungsleiterin Daten, Demographie und Qualität DDQ 